\title{
Numerical approximation of the effective Hamiltonian and of the Aubry set for first order Hamilton-Jacobi equations
}

\author{
Marco Rorro* \\ Dipartimento di Metodi e Modelli Matematici \\ Università di Roma "La Sapienza" \\ E-mail: rorrodcaspur.it
}

\begin{abstract}
We introduce an approximation for first order Hamilton-Jacobi equations with a convex Hamiltonian periodic in the space variable. We use a first order semi-Lagrangian scheme to compute a solution of the so called cell problem which allows us to compute the effective Hamiltonian. We exploit the information included in the solutions of the cell problem and in the effective Hamiltonian to obtain an approximation of the Aubry set. These objects are relevant from a PDE as well as from a dynamical point of view. Two numerical tests illustrate the effectiveness of our approximation.
\end{abstract}

Control Systems: Theory, Numerics and Applications

30 March - 1 April 2005

Rome

* Speaker. 


\section{Introduction}

This paper deals with the numerical solution of the so called cell-problem for a periodic Hamilton-Jacobi equation

$$
H\left(x, D u+p_{0}\right)=c \text { on } \mathbb{T}^{n}
$$

where $\mathbb{T}^{n}=\mathbb{R}^{n} / \mathbb{Z}^{n}$ is the $n$-th dimensional torus, $p_{0} \in \mathbb{R}^{n}$ and $c \in \mathbb{R}$. We study, for any fixed $p_{0}$, the numerical approximation of the critical value $\bar{H}\left(p_{0}\right)$, i.e. the unique value of $c$ such that the corresponding equation (1.1) has a (viscosity [2, 1]) solution. In general this solution is not unique on the torus $\mathbb{T}^{n}$ see [21, 9]. The function $\bar{H}\left(p_{0}\right)$ is known as the effective Hamiltonian. Our numerical experiments deal with the eikonal equation

$$
\left|D u+p_{0}\right|=f(x)+c .
$$

Even in this simple setting an explicit solution of (11.1) is available only for particular functions $f$. Other numerical approximations have been proposed in the last years. The first one was proposed by Gomes and Oberman in [17]. It is based on a discrete version of a min-max formula due to [7]:

$$
\bar{H}\left(p_{0}\right)=\inf _{\varphi \in C_{p e r}^{1}}\left(\mathbb{T}^{n}\right) \sup _{x \in \mathbb{T}^{n}} H\left(p_{0}+D \varphi, x\right)
$$

where the infimum is taken over the space $C^{1}\left(\mathbb{T}^{n}\right)$ of periodic function. The numerical approximation is based on a $\mathbb{P}_{1}$ approximation of periodic functions on a uniform structured grid on the torus. Denoting by $P_{h}$ ( $h$ is the space step) the piecewise linear finite elements space, the discrete problem is

$$
\bar{H}\left(p_{0}\right)=\inf _{\varphi_{h} \in P_{h}} \sup _{x \in \mathbb{T}^{n}} H\left(p_{0}+D \varphi, x\right)
$$

The main disadvantage of this method is that the computation at every $p$ relies on a min-max problem in a high-dimensional space $\left(\mathbb{R}^{d}\right.$ where $d$ is the number of nodes in the grid) and this produces a rather slow algorithm. Moreover, the search of the minimum, made by a SQP (Sequential Quadratic Programming) algorithm, provides only the value of $\bar{H}$. No information on the solution of the cell problem is obtained.

In a more recent work due to Qian [22] a monotone finite difference scheme is used to approximate two related problems:

$$
\alpha u+H\left(x, D u+p_{0}\right)=c \text { on } \mathbb{T}^{n}
$$

and

$$
u_{t}+H\left(x, D u+p_{0}\right)=0 \text { on } \mathbb{T}^{n}
$$

In the first case is known, by the results in [21], that

$$
\bar{H}\left(p_{0}\right)=-\lim _{\alpha \rightarrow 0} \alpha u^{(\alpha)} .
$$

In the second case Qian exploits the relation

$$
\bar{H}\left(p_{0}\right)=-\lim _{t \rightarrow+\infty} \frac{u(x, t)}{t} .
$$


It is worth noting that in this last case it is possible to find an approximation of $\bar{H}$ also if $u(x, t)$ does not converge to a solution of the cell problem. In this paper, we use a similar approach, but our first objective is to look for a solution of the cell problem (1.1). From this solution we get an approximation of $\bar{H}$, as well as additional information on the so called (projected) Aubry set $\mathcal{A} \subseteq \mathbb{T}^{n}$. This set is a uniqueness set for (1.1), i.e. every solution which coincides on $\mathcal{A}$, coincides everywhere. To approximate this set we follow the backward characteristics. A second approach for the approximation of this set is to compare two different subsolutions. So we consider the symmetrical problem relatet to (1.1)

$$
H\left(x, D u-p_{0}\right)=c \text { on } \mathbb{T}^{n} .
$$

This equation has the same critical value of (1.1) and if $\check{u}$ is its solution, then $-\check{u}$ is a subsolution of (1.1), see [15].

We use a semilagrangian scheme, [11, 12], to approximate a solution of (1.1) and (1.8).

The convergence can be established thanks to a result of Ishikawa [18] as in [5, 6].

The paper is organized as follow.

In Section 2 some basic results of the continuous problem are shown. Section 3 is devoted to the discrete version of the continuous problem and to the proof of convergence of the numerical scheme. Finally, in Section 4 we present our numerical tests in 1D and 2D.

\section{Basic results on the continuous problem}

In this section we describe some basic results on the continuous problem, that we report here to introduce the problem. A detailed description can be found in [14, 15, 5, 5. We consider the initial value problem

$$
\begin{cases}u_{t}+H(x, D u)=0 & \text { in } \left.\mathbb{T}^{n} \times\right] 0,+\infty[ \\ u(x, 0)=u_{0}(x) & \text { in } \mathbb{T}^{n} \times\{0\}\end{cases}
$$

whose solution can be represented by the so called Lax-Hopf-Oleinik nonlinear operator

$$
T_{t} u_{0}(x)=\inf _{\gamma}\left\{u_{0}(\gamma(0))+\int_{0}^{t} L(\gamma, \dot{\gamma}) d s\right\}
$$

where the infimum is taken over all the absolutely continuous curves $\gamma:[0, t] \rightarrow \mathbb{T}^{n}$ such that $\gamma(t)=$ $x$. The Lagrangian $L$ is the Fenchel transform of $H$, i.e.

$$
L(x, q)=\sup _{p \in \mathbb{R}^{n}}\{p q-H(x, p)\} .
$$

$H$ is supposed at least bounded below and continuous in both variables, convex and coercive in $p$. If $u_{0}(x)$ is a solution of the cell problem (1.1), then the following result holds.

Proposition 2.1. [21, 9 (4) For each fixed $p_{0} \in \mathbb{R}^{n}$, if $u$ is a solution of the cell problem (1.1] with $c=\bar{H}\left(p_{0}\right)$ then for each $t>0$

$$
T_{t} u(x)=u(x)-\bar{H}\left(p_{0}\right) t
$$


For a fixed time $t>0$, the problem (2.4) is an (additive nonlinear) eigenvalue problem, that is, to find eigenfunctions $u$ and (additive) eigenvalues $\lambda=-\bar{H}\left(p_{0}\right) t$ such that $T u=u+\lambda$, where

$$
T u(x)=\min _{y \in \mathbb{R}^{n}}\{u(y)+K(x, y)\}
$$

and

$$
K(x, y)=\inf _{\gamma}\left\{\int_{0}^{t} L(\gamma, \dot{\gamma}) d s\right\}
$$

now the infimum is taken over all the absolutely continuous curves $\gamma:[0, t] \rightarrow \mathbb{T}^{n}$ such that $\gamma(0)=y$ and $\gamma(t)=x$. It is worth noting that such function is not necessarily unique whereas the eigenvalue is unique and independent on $t$. Once an eigenfunction $u$ has been computed, we can recover $\bar{H}$ by the following formula

$$
\bar{H}\left(p_{0}\right)=-\frac{T_{t} u-u}{t} .
$$

\section{The discrete problem}

Let us fix a time step $\Delta t=h$ and write

$$
T_{h} u(x)=\min _{y \in \mathbb{R}^{n}}\left\{u(y)+K_{h}(x, y)\right\}
$$

with

$$
K_{h}(x, y)=\inf _{\gamma}\left\{\int_{0}^{h} L(\gamma, \dot{\gamma}) d s\right\}
$$

where the infimum is taken over all the absolutely continuous curves $\gamma:[0, h] \rightarrow \mathbb{T}^{n}$ such that $\gamma(0)=y$ and $\gamma(h)=x$. For $h$ sufficiently small we have the following discrete eigenvalue problem

$$
u(x)=T_{h} u(x)+\lambda h \text { on } \mathbb{T}^{n}
$$

where

$$
T_{h} u(x)=\inf _{a \in \mathbb{R}^{n}}\{u(x+a h)+L(x, a) h\} .
$$

The discrete problem (3.4), or discrete Hamilton-Jacobi equation, has been studied also in the recent work of Gomes [16] as a dual problem of an infinite dimensional linear programming problem on a space of measure, called a discrete version of the Aubry-Mather problem. Usually, such problem is approximated by an ergodic operator,

$$
T_{h}^{\alpha} u(x)=e^{-\alpha} \inf _{a \in \mathbb{R}^{n}}\{u(x+a h)+L(x, a) h\}
$$

for $\alpha>0$. In this way, $T_{h}^{\alpha}$ is a strict contraction, and its convergence to the unique fixed point can be easily proved. This paper deals with the case $\alpha=0$, so $T_{h}$ is not a strict contraction but is only nonexpansive.

Proposition 3.1. Let $T_{h}$ as in (3.4), then

1. $\left\|T_{h} u(x)-T_{h} v(x)\right\|_{\infty} \leq\|u(x)-v(x)\|_{\infty} ;$ 
2. $T_{h} u \leq T_{h} v$ for any $u \leq v$;

3. $T_{h}[u+c]=T_{h} u+c$ for any $c \in \mathbb{R}$.

\section{Proof.}

1. For any $u, v$, let $a^{*}(x)$ such that $v\left(x+a^{*}(x) h\right)+L\left(x, a^{*}(x)\right) h=\inf _{a \in \mathbb{R}^{n}}\{v(x+a h)+L(x, a) h\}$,

$$
\begin{aligned}
T_{h} u(x)-T_{h} v(x) & \leq u\left(x+a^{*}(x) h\right)+L\left(x, a^{*}(x)\right) h-v\left(x+a^{*}(x) h\right)-L\left(x, a^{*}(x)\right) h \\
& =u\left(x+a^{*}(x) h\right)-v\left(x+a^{*}(x) h\right) \leq\|u(x)-v(x)\|_{\infty}
\end{aligned}
$$

then $\left\|T_{h} u(x)-T_{h} v(x)\right\|_{\infty} \leq\|u(x)-v(x)\|_{\infty}$.

The two other points can be easily obtained by the definition of $T_{h}$.

The periodic assumption on the space variable $x$ plays a key role in the next development. In this case, $T_{h} u$ is periodic in $x$. Moreover,

$$
T_{h} u(x)=\inf _{a \in \mathbb{R}^{n}}\{u(x+a h)+\tilde{L}(x, a) h\}
$$

where

$$
\tilde{L}(x, a)=\inf _{k \in \mathbb{Z}^{n}}\{L(x, a+k)\} .
$$

$\tilde{L}$ is periodic in both variables, so $T_{h} u$ is bounded, and being $T u$ continuous from the nonexpansivennes, we get that $T_{h}$ is uniformly continuous.

Let us define the Banach space

$$
X=\left\{[u]: u \in C\left(\mathbb{T}^{n}\right), v \sim \tilde{v} \Longleftrightarrow v-\tilde{v}=\text { cost } .\right\}
$$

and the closed subset

$$
D=\left\{u \in X:\|u\|_{\infty} \leq M\right\}
$$

Clearly $T_{h}(X) \subset X$.

Proposition 3.2. $T_{h}(D)$ is precompact.

Proof. We check that $T_{h}(D)$ is equicontinuous and uniformly bounded, so that by the AscoliArzela's theorem we get that $T_{h}(D)$ is precompact. $T_{h}(D)$ is uniformly bounded. Indeed, for any $u \in D$

$$
\left\|T_{h} u\right\|_{\infty} \leq\|u(x)+L(x, 0)\|_{\infty} \leq M+C h .
$$

Let us prove that $T_{h}(D)$ is equicontinuous. In fact, for any $u \in D$, let $z^{\prime}$ such that

$$
\inf _{a \in \mathbb{R}^{n}}\{u(z+a h)+L(y,(z-y) / h) h\}=u\left(z^{\prime}\right)+L\left(y,\left(z^{\prime}-y\right) / h\right) h
$$

then

$$
\begin{aligned}
T_{h} u(x)-T_{h} u(y) & \leq \inf _{z \in \mathbb{R}^{n}}\{u(z)+L(x,(z-x) / h) h\}-u\left(z^{\prime}\right)-L\left(y,\left(z^{\prime}-y\right) / h\right) h \\
& \leq u\left(z^{\prime}\right)+L\left(x,\left(z^{\prime}-x\right) / h\right) h-u\left(z^{\prime}\right)-L\left(y,\left(z^{\prime}-y\right) / h\right) h \\
& =L\left(x,\left(z^{\prime}-x\right) / h\right) h-L\left(y,\left(z^{\prime}-y\right) / h\right) h
\end{aligned}
$$

so that the equicontinuity of $T_{h}$ comes from the uniform continuity of $L$ or, equivalently, of $\tilde{L}$. 
The following theorem of Ishikawa, an extension of the Schauder's theorem, gives the existence of a fixed point for $T_{h}$, and a constructive way to obtain an approximation.

Theorem 3.3 (Ishikawa's) Let $D$ be a closed subset of a Banach space $X$ and let $S$ be a nonexpansive mapping from $D$ into a compact subset of $X$. If there exists $\tau \in(0,1)$ such that $(1-\tau) u+\tau S u \in$ $D$ for all $u \in D$, then $S$ has a fixed point in $D$ and for any $u^{0} \in D$, the sequence defined inductively by $u^{n+1}=(1-\tau) u^{n}+\tau S u^{n}$ converges to a fixed point of $S$.

So the following scheme

$$
(1-\tau) u+\tau T_{h} u=\inf _{a \in \mathbb{R}^{n}}\{(1-\tau) u(x)+\tau u(x+a h)+L(x, a) \tau h\}
$$

defines a sequence $\left\{u^{n}\right\}$ which converges to an (additive) eigenfunction of $T_{h}$. In the piecewise finite element space $P_{h}$, for $h$ sufficently small, we get

$$
(1-\tau) u(x)+\tau u(x+a h)=u(x+a \tau h)
$$

so that the previous scheme coincides with $T_{\tau h}$, and by (2.7)

$$
\bar{H}\left(p_{0}\right)=-\lim _{n \rightarrow+\infty} \frac{u^{n+1}-u^{n}}{\tau h} .
$$

The solution $u$ contains also information on its uniqueness set that we get following the discrete backward characteristic

$$
\left\{\begin{array}{l}
x_{n+1}=x_{n}+a^{*}\left(x_{n}\right) \tau h \\
x_{0}=x
\end{array}\right.
$$

where $a^{*}\left(x_{n}\right)$ is such that

$$
\inf _{a \in \mathbb{R}^{n}}\left\{(1-\tau) u\left(x_{n}\right)+\tau u\left(x_{n}+a h\right)+L\left(x_{n}, a\right) \tau h\right\}=(1-\tau) u\left(x_{n}\right)+\tau u\left(x_{n}+a^{*} h\right)+L\left(x_{n}, a^{*}\left(x_{n}\right)\right) h \tau
$$

This is also the synthesis of the optimal feedback control for the value function $u$ in control theory, see [11], and the optimal path in [5]. In this way, we can recover one component of the set $\mathcal{A}$. For a complete understanding of such set, it is useful to study also the symmetrical problem

$$
H\left(x, D u-p_{0}\right)=c
$$

and the relation between the solution of this problem with the solution of the original one. This part is still under development. We will present some numerical experiments in the next section and we refer to [13] for theoretical results.

\section{Numerical results}

In this section we consider the following problem

$$
\left|D u+p_{0}\right|=\sqrt{2(f(x)+c)}
$$


in one and two dimensions. We solved it in the piecewise finite element space $P_{h}$ by the scheme (3.8) that in this case becomes

$$
\inf _{a \in B(0,1)}\{(u(x+a \tau h)+L(x, a) \tau h\}
$$

where $B(0,1)$ is the unit ball. In one dimension we discretize it by the set $\{-1,0,1\}$. In two dimensions we consider the set $\{0, \partial B\}$ and the search of the minimum on the boundary of $B$ is made by bisection. The scheme used is part of the public domain HJPACK library, [20].

The parameter $\tau$ in (4.2) is usually fixed at 0.5. A numerical example with $\tau=1$ which reproduces the transport phenomena of the analytical counter-example in [3, 8] can be found in [23].

For each $p_{0}$ we stop the scheme when $\left\|u^{n+1}-u^{n}\right\|_{\infty}<\varepsilon$.

\section{Test 1}

Let us consider problem (4.1) approximated by (4.2) with $h=0.01$ and

$$
f(x)=\frac{1}{5} \sin ^{2}(2 \pi x)(1-\cos (2 \pi x)) .
$$

The same problem has been considered with a different approach also in [19].

Figure 11 shows the computed $\bar{H}$. In this case it is known that $\bar{H}\left(p_{0}\right)=0$ if $\left|p_{0}\right|<\int_{0}^{1} \sqrt{2 f(x) \mathrm{d} x} \simeq$ 0.3982 (compare it with the right of Figure 1). Otherwise, $\bar{H}\left(p_{0}\right)$ satisfies

$$
\left|p_{0}\right|-\int_{0}^{1} \sqrt{2\left(f(x)+\bar{H}\left(p_{0}\right)\right) \mathrm{d} x}=0 .
$$
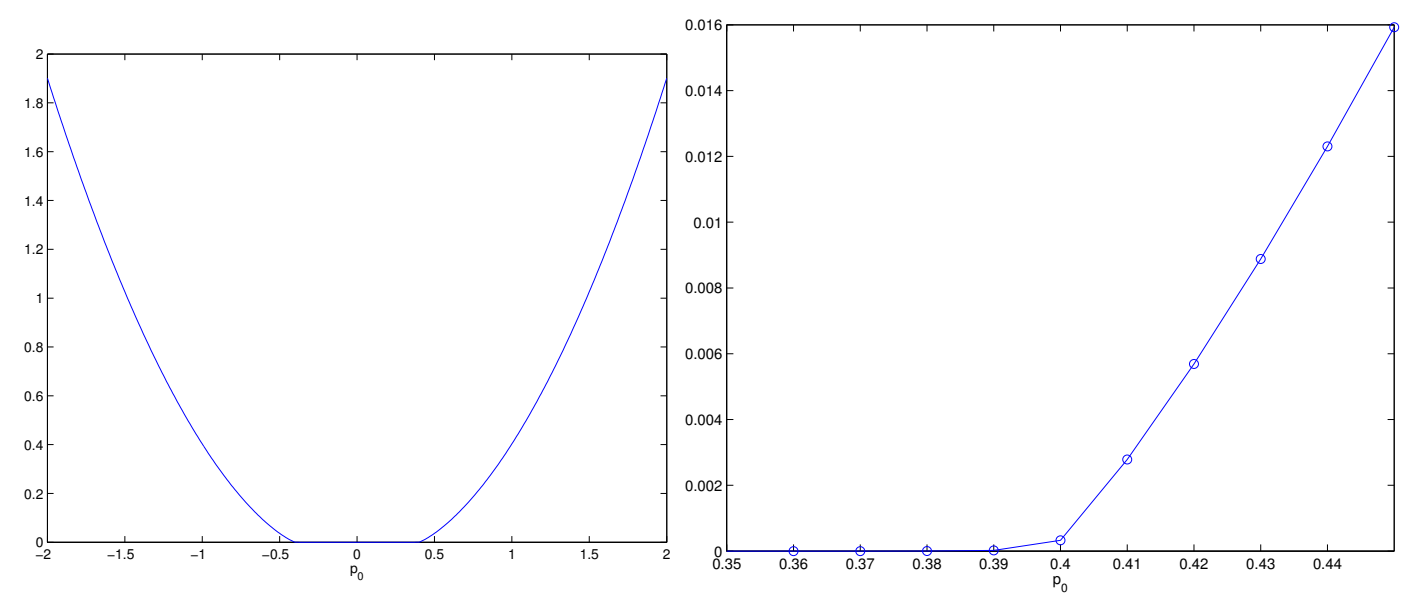

Figure 1: $\bar{H}\left(p_{0}\right)$ computed in $[-2,2]$ every $0.01, \varepsilon=10^{-8}, h=0.01$

If $p_{0}$ is in the flat part of $\bar{H}\left(\left|p_{0}\right| \lesssim 0.39\right)$ then the Aubry set is composed only by two points, $x=0$ and $x=0.5$. If $p_{0}$ is out of the flat part of $\bar{H}$, then the Aubry set is the whole torus.

Three different solutions at the equilibrium level, that is for $p_{0}$ in the flat part of $\bar{H}$, are shown on the left of Figure 2. From $u_{1}$ and $u_{3}$, many other solutions like $u_{2}$ can be obtained. Note that fixed the value zero of the solution at $x=0$, there exists a solution for any choice of its value approximately between $[-0.2,0.2]$ at $x=0.5$ and that the length of such interval is almost exactly the distance 
between $p_{0}$ and the boundary of the flat part of $\bar{H}$. On the right of Figure \&, we have plotted the derivative of the solutions on the left (computed by centered difference) and the contour levels of $\widetilde{H}(x, D u)=\frac{1}{2}|D u|^{2}-f(x)$, that is the Hamiltonian flow. The zero contour level of $\widetilde{H}$ coincides with the zero contour level of $H(x, D u)=|D u|-\sqrt{2 f}$.
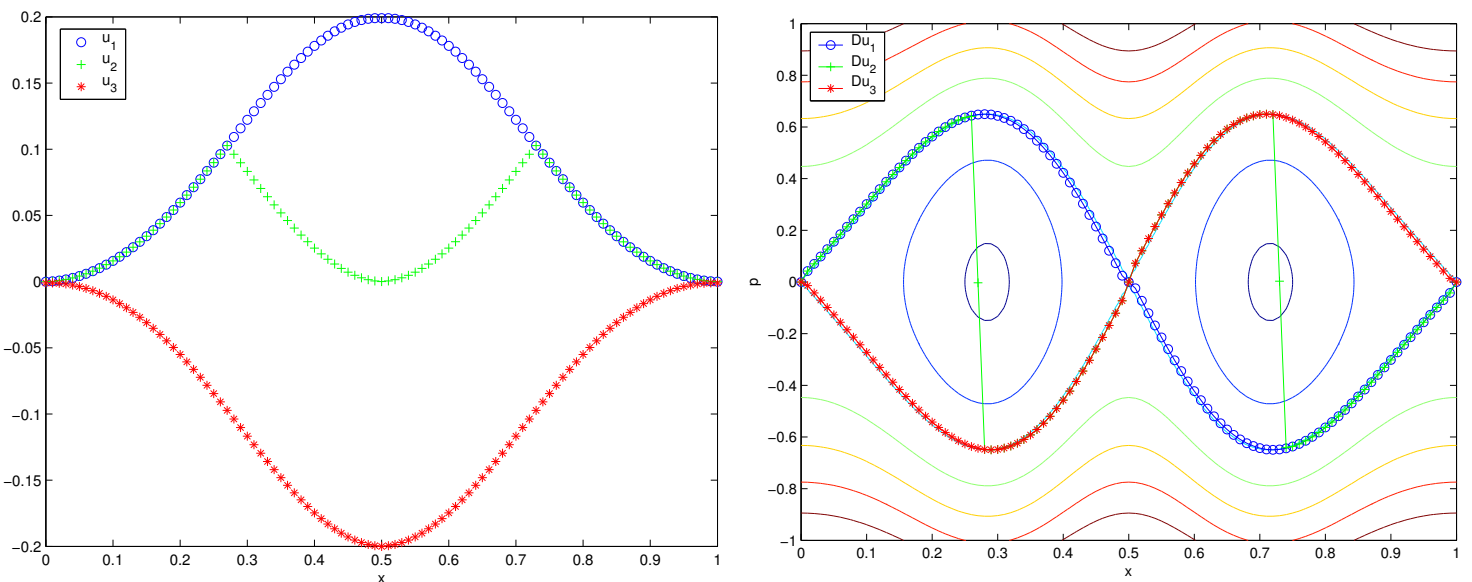

Figure 2: Three solutions for $p_{0}=0$, starting from different $u_{0},-\cos (2 \pi x)+1,0, \cos (2 \pi x)+0.8$ respectively on the left. Their derivative and the contour levels of $\tilde{H}$ on the right

Figure 3 shows different solutions for $p=0.2$. If we fix its value at zero in the origin, the range of its possible values at $x=0.5$ reduces approximately to $[-0.1,0.1]$, whose length is again the distance of $p_{0}$ from the boundary of the flat part of $\bar{H}$. This can be seen also in the same solutions plus $p_{0} x$ in the right of Figure 3 . The characteristics are again backward invariant tori of the Hamiltonian flow as in the previous case.
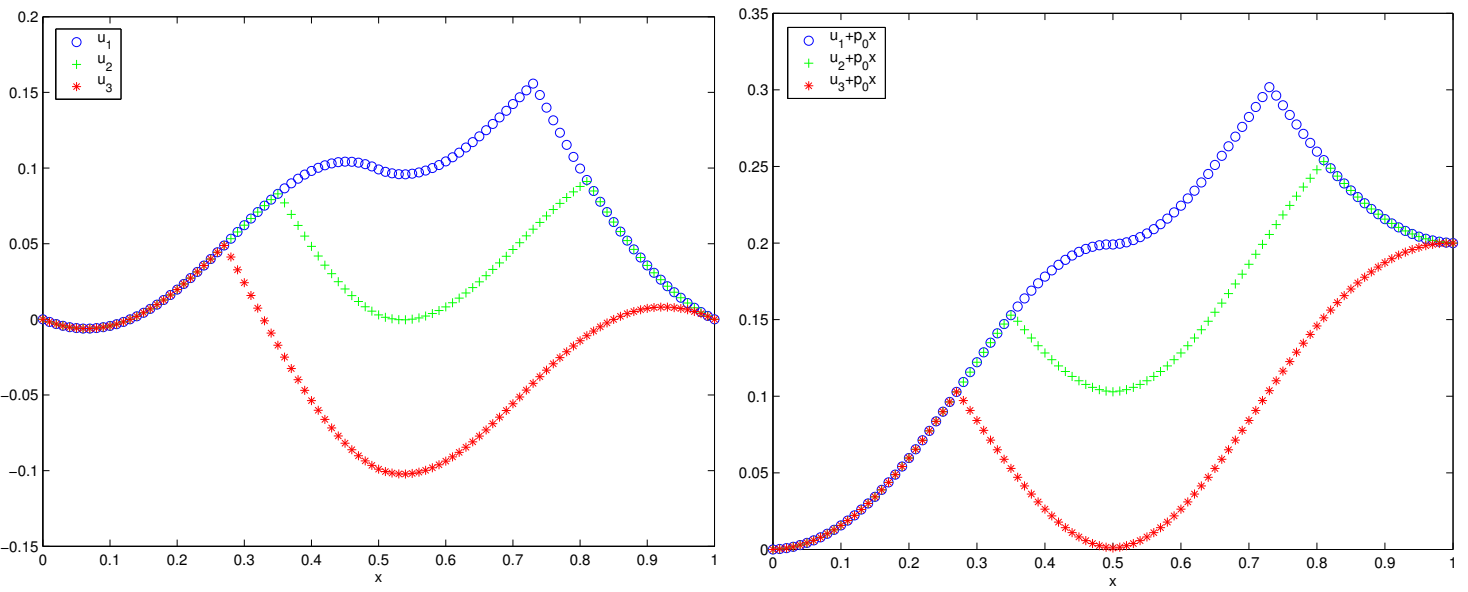

Figure 3: Three solutions for $p_{0}=0.2$, starting from different $u_{0},-\cos (2 \pi x)+1,0, \cos (2 \pi x)+0.8$ respectively and shifted to coincide at the origin on the left. On the right the same solutions plus $p_{0} x$.

Figure 4 shows two solutions for two different values of $p_{0}$ out the flat part of $\bar{H}$. In this case the solution is unique for each fixed $p_{0}$. The $\bar{H}\left(p_{0}\right)$ contour level of $\widetilde{H}$ coincides with the zero contour level of $H(x, D u)=|D u|-\sqrt{2\left(f+\bar{H}\left(p_{0}\right)\right)}$ which are invariant tori. 

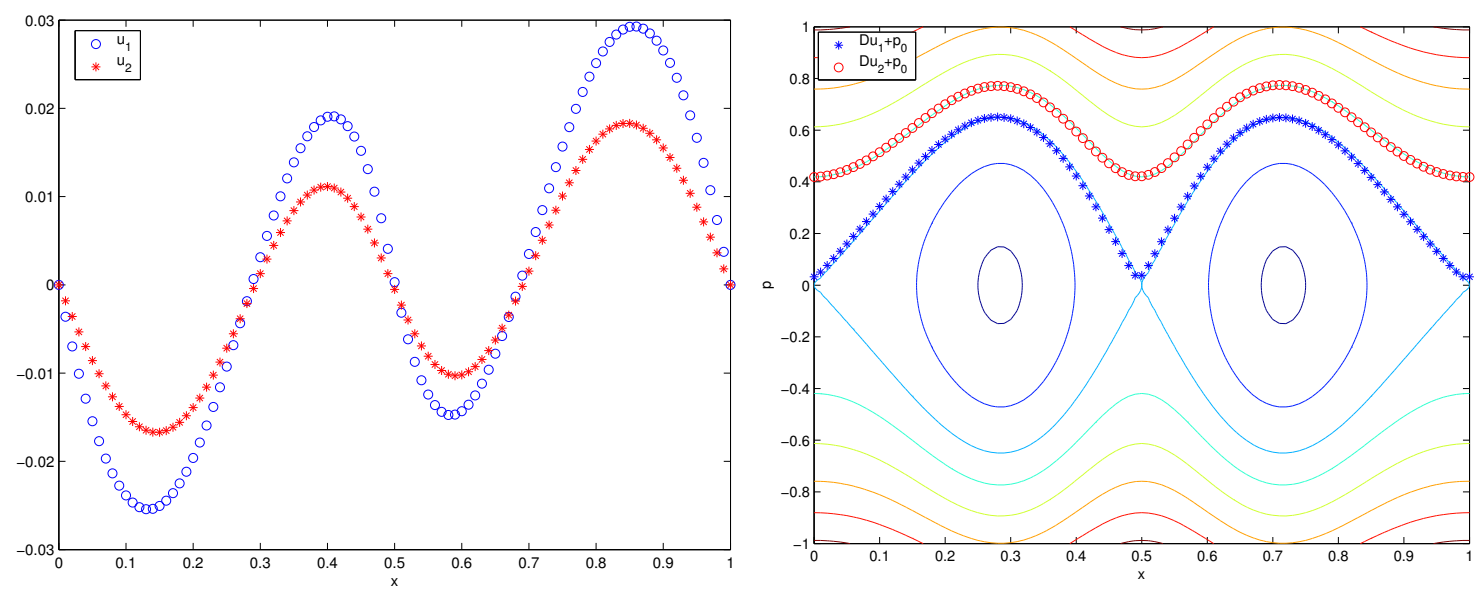

Figure 4: Two solutions, $u_{1}$ for $p_{0}=0.4$ and $u_{2}$ for $p_{0}=0.6$ shifted to coincide at the origin on the left. Their derivative plus $p_{0}$ and the contour levels of $\tilde{H}$ on the right

\section{Test 2}

This test, in two dimensions, considers equation (4.1), with

$$
f(x)=\cos \left(2 \pi x_{1}\right)+\cos \left(2 \pi x_{2}\right)+\cos \left(2 \pi\left(x_{1}-x_{2}\right)\right) .
$$

Figure 5 shows the computed $\bar{H}$ and its contour levels. The same test is also in [17, 10] (with which Figure 5 can be compared). The contour levels of $\bar{H}$, on the right of Figure 5 , shows some regions where one of the partial derivatives of $\bar{H}$ is zero (see also Figure 6 ) and other regions in which the partial derivatives are equal. In both cases they are rationally dependent. Between these regions, the partial derivatives vary with continuity. We concentrate our test on the approximation of the set $\mathcal{A}$ for values of $p_{0}$ in one of this last regions. In fact, only in these regions the value of $D \bar{H}$ can be rationally independent, and so the phenomena of characteristic of infinite length can happen, see [24] and Figure 8 and 11. Together with the computed characteristics, we visualize $u+\breve{u}$, where $\check{u}$ indicates the solution of the symmetrical problem (1.8). It is noteworthy that in these regions the set $\mathcal{A}$ as function of $p_{0}$ is discontinuous, as the sequence of Figures from 7 to 11 shows.
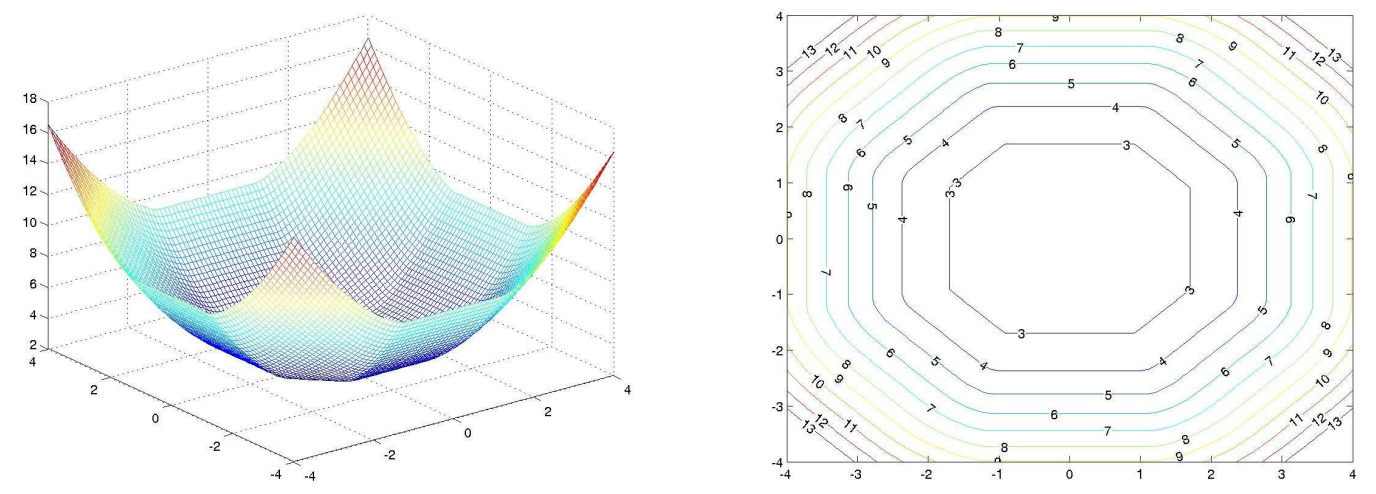

Figure 5: $\bar{H}\left(p_{0}\right)$ computed in $[-4,4]$ every $0.1, \varepsilon=10^{-6}, h=0.05$ 

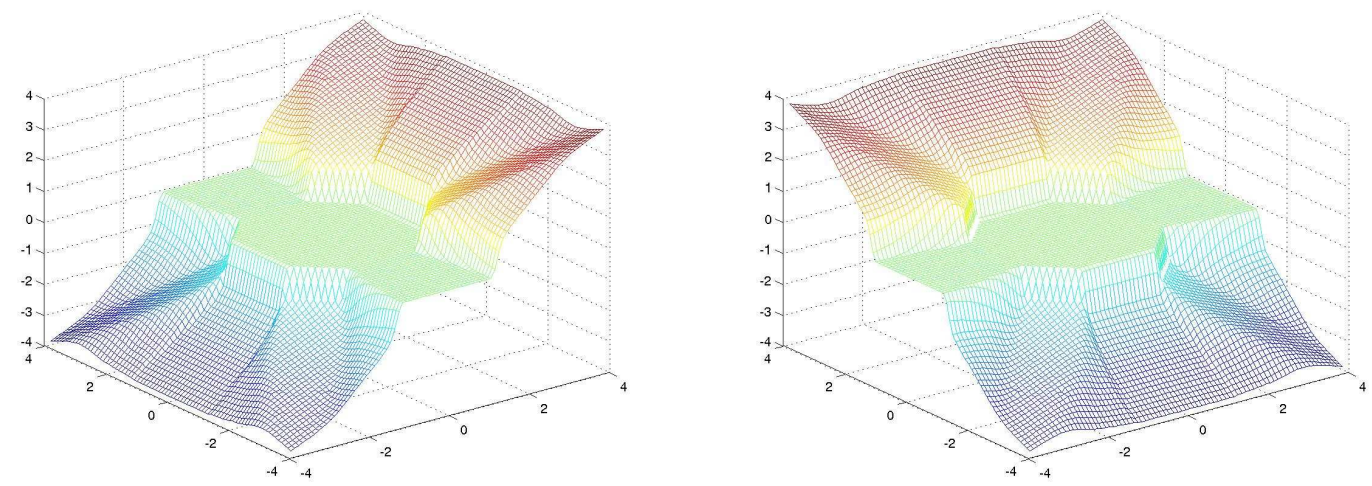

Figure 6: $D \bar{H}\left(p_{0}\right)$ computed by centered difference
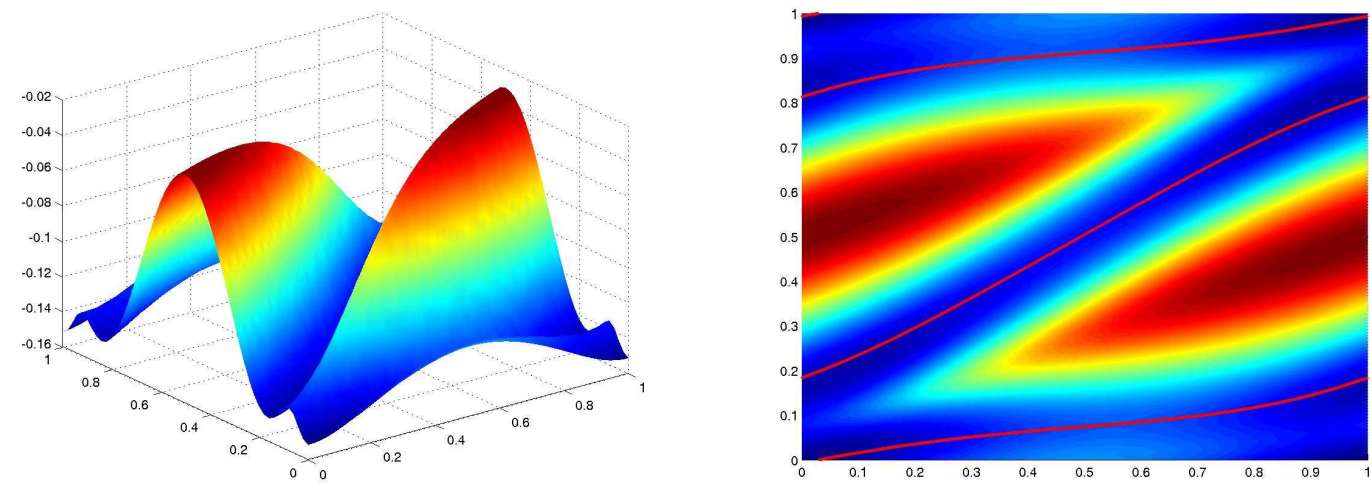

Figure 7: $u+\check{u}$ for $\varepsilon=10^{-6}, h=0.02, p_{0}=(3.8,1.4), \bar{H} \simeq 8.4780$

\section{References}

[1] M. Bardi and I. Capuzzo Dolcetta, Optimal Control and Viscosity Solutions of Hamilton-Jacobi Bellman equations, Birkhäuser, Boston, 1994.

[2] G. Barles, Solutions de viscosité des equations de Hamilton-Jacobi, Mathematics and Applications, Springer - Verlag, 1994.

[3] G. Barles and P.E. Souganidis, Convergence of approximation schemes for fully nonlinear second order equations, Asymptotic Analysis 4, (1991), 271-283.

[4] G. Barles and J.M. Roquejoffre, Large time behaviour of fronts governed by eikonal equations, Interfaces and Free Boundaries 5 (2003), 83-102.

[5] M.C. Concordel, Periodic Homogenization of Hamilton-Jacobi Equations, Ph.D. Thesis, UC Berkeley, (1995).

[6] M.C. Concordel, Periodic homogenization of Hamilton-Jacobi equations: Additive eigenvalues and variational formula, Indiana Univ. Math. J. 45 (1996), 1095-1117.

[7] G. Contreras, R. Iturriaga, G.P. Paternain, M. Paternain, Lagrangian graphs, minimizing measures and Mañé's critical values, Geom. Funct. Anal. 8 (1998), 788-809. 

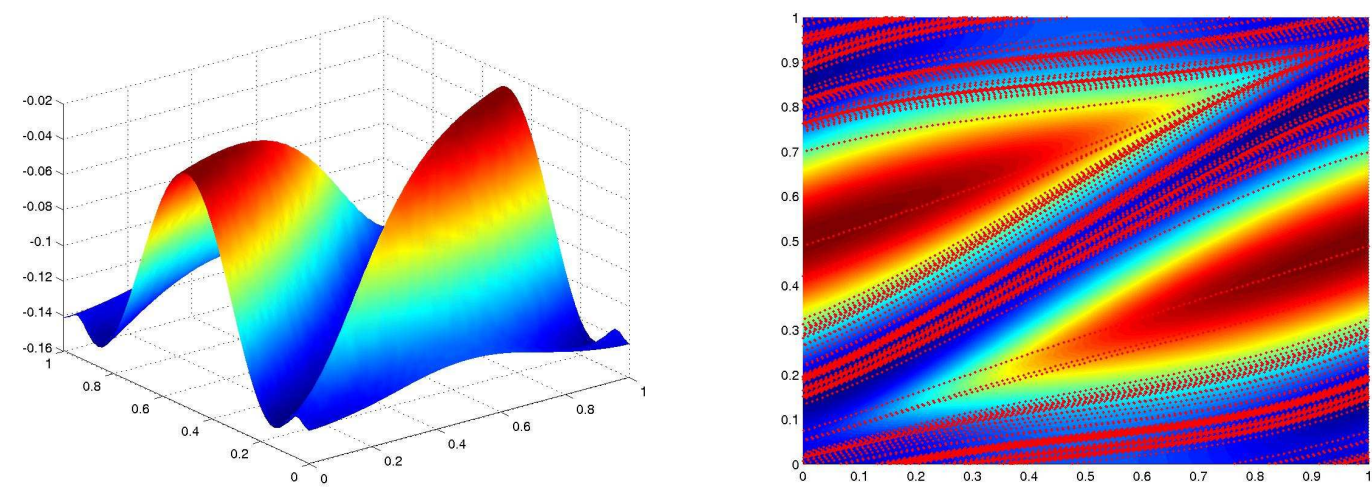

Figure 8: $u+\check{u}$ for $\varepsilon=10^{-6}, h=0.02, p_{0}=(3.8,1.5), \bar{H} \simeq 8.6133$
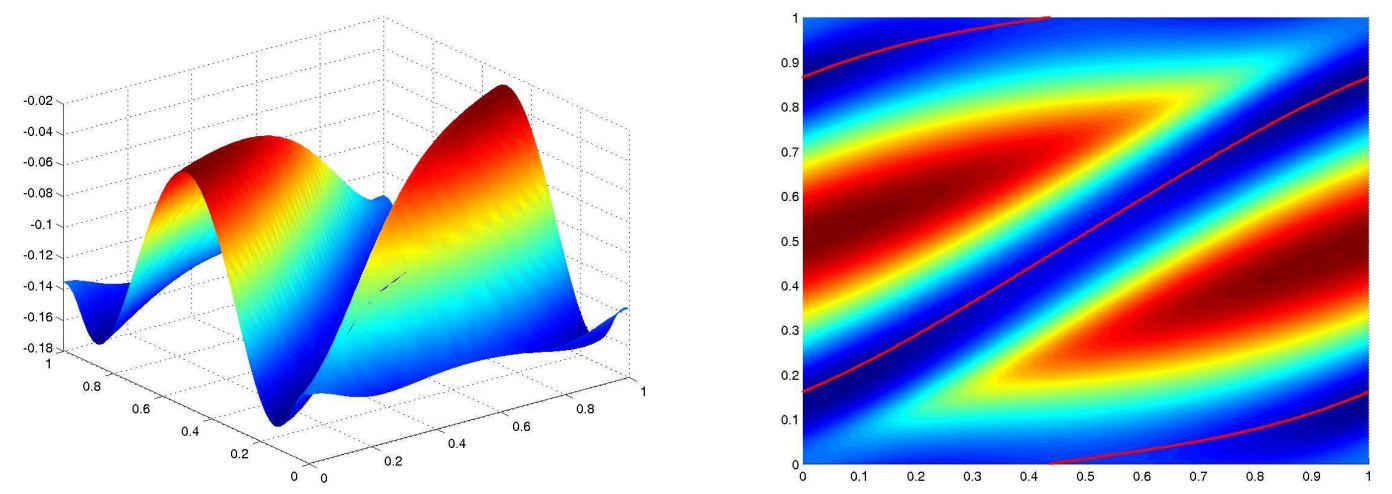

Figure 9: $u+\check{u}$ for $\varepsilon=10^{-6}, h=0.02, p_{0}=(3.8,1.6), \bar{H} \simeq 8.7667$

[8] A. Davini and A. Siconolfi, A Generalized Dynamical Approach to the Large time behavior of solutions of Hamilton-Jacobi equations, preprint, (2005).

[9] L.C. Evans, Periodic homogenization of certain nonlinear partial differential equation, Proc. Roy. Soc. Edinburgh, 120 A, (1992) 245-265.

[10] L.C. Evans, A survey of partial differential equations methods in weak KAM theory, Communication in Pure and Applied Mathematics 57 (2004), 445-480.

[11] M. Falcone, Numerical solution of dynamic programming equations, Birkhäuser, Boston, 1994, Appendix A, in [1].

[12] M. Falcone and R. Ferretti, Semi-Lagrangian schemes for Hamilton-Jacobi equations, discrete representation formulae and Goudonov methods, J. Comput. Phys. 175 (2002), 559-575.

[13] M. Falcone, M. Rorro, A. Siconolfi, Numerical approximation of the effective Hamiltonian and of the Aubry set, in preparation.

[14] A. Fathi, Weak KAM Theorem in Lagrangian Dynamics, Cambridge University Press, 2004.

[15] A. Fathi and A. Siconolfi, PDE aspects of Aubry-Mather theory for quasiconvex Hamiltonians, Calc. Var. Partial Differential Equations 22 (2005), 185-228. 

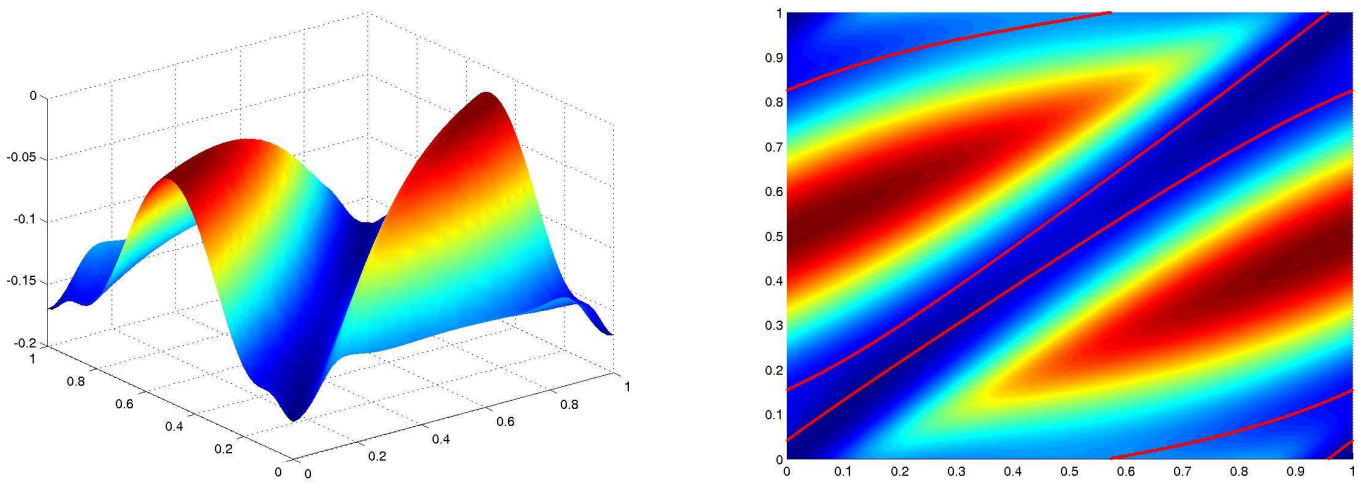

Figure 10: $u+\check{u}$ for $\varepsilon=10^{-6}, h=0.02, p_{0}=(3.8,2.1), \bar{H} \simeq 9.7177$
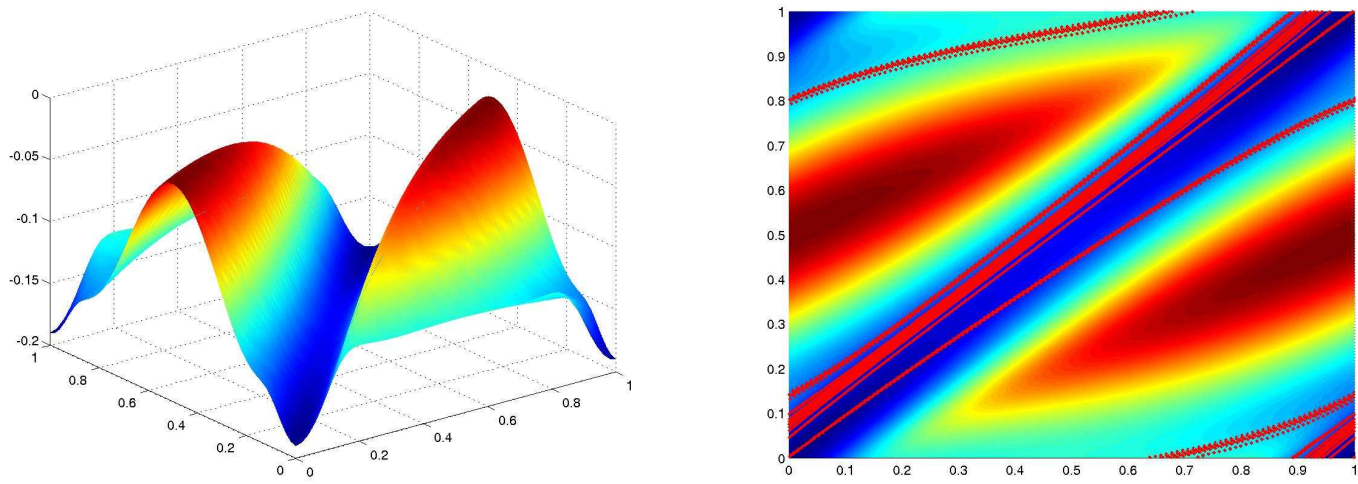

Figure 11: $u+\check{u}$ for $\varepsilon=10^{-6}, h=0.02, p_{0}=(3.8,2.2), \bar{H} \simeq 9.9553$

[16] D.A. Gomes, Viscosity solution methods and the discrete Aubry-Mather problem, Discrete and continuous dynamical system, 13 (2005) 103-116.

[17] D.A. Gomes and A. Oberman, Computing the effective Hamiltonian using a variational approach, SIMA J. Cont. Opt. 43 (2004), 798-812.

[18] S. Ishikawa, Fixed points and iteration of a nonexpansive mapping in a Banach space, Proc. Am. Math. Soc. 59 (1976), 65-71.

[19] H.R. Jauslin, H.O. Kreiss and J. Moser, On the forced Burgers equation with periodic boundary conditions, Proceedings of Symposia in Pure Mathematics 65 (1999), 133-153.

[20] http://wWw. caspur.it/hjpack

[21] P.L. Lions, G. Papanicolau and S.R.S. Varadhan, Homogenization of Hamilton-Jacobi equations, unpublished preprint, (1987).

[22] J. Qian, Two approximations for effective Hamiltonians arising from homogenization of Hamilton-Jacobi equations, UCLA, Department of Mathematics, preprint, (2003).

[23] M. Rorro, An approximation scheme for the effective Hamiltonian and applications, to appear on Applied Numerical Mathematics, (2005). 
[24] Weinan E, Aubry-Mather theory and periodic solutions of the forced Burgers equation, Communication in Pure and Applied Mathematics 52 (1999), 811-828 